\title{
Coherent Zone-Folded Longitudinal Acoustic Phonons in Semiconductor Superlattices: Excitation and Detection
}

\author{
Albrecht Bartels,* Thomas Dekorsy, and Heinrich Kurz \\ Institut für Halbleitertechnik II, Rheinisch-Westfälische Technische Hochschule Aachen, \\ Sommerfeldstrasse 24, D-52056 Aachen, Germany \\ Klaus Köhler \\ Fraunhofer-Institut für Angewandte Festkörperphysik, D-79108 Freiburg, Germany
}

\begin{abstract}
Coherent zone-folded acoustic phonons are excited in GaAs/AlAs superlattices by femtosecond laser pulses via resonant impulsive stimulated Raman scattering in both forward and backward scattering directions. The relative amplitudes of three distinct modes of first and second backfolded order match well with scattering intensities calculated within an elastic continuum model. The detection of the coherent acoustic modes is based on the modulation of the interband transitions via the acoustic deformation potential and exhibits a strong enhancement at interband transitions.
\end{abstract}

The investigation of low-energy elementary excitations in semiconductor heterostructures is driven by their relevance as the final state in the energy relaxation process. In particular, acoustic phonons play a dominant role for dephasing processes at low lattice temperature and for heat transport in general. Acoustic phonons are commonly investigated by continuous wave (cw) Brillouin scattering. Time resolved experiments based on ultrashort pulse lasers have largely contributed to the understanding of acoustic phonon dynamics. Recently, the generation and propagation of ballistic acoustic phonons in a single semiconductor quantum well was observed by surface deflection spectroscopy [1]. Semiconductor superlattices exhibit zone folding of the acoustic branches within the mini-Brillouinzone (mini-BZ) due to the artificial periodicity of the elastic properties along the growth direction. Here, light can couple to zone-folded acoustic modes of the superlattice at higher frequencies in the $100 \mathrm{GHz}$ to $\mathrm{THz}$ range. These modes have been extensively studied in cw Raman spectroscopy [2-4]. The folded bulk acoustic branches are optical branches within the superlattice zone scheme; thus light scattering from those modes is referred to as Raman scattering. The coherent excitation of a single first-order zone-folded mode was observed in GaAs/AlAs superlattices using a time-derivative detection scheme [5]. However, the excitation and detection mechanisms are not yet fully clarified. In this paper we report on the nature of the excitation and detection mechanisms relevant for coherent zone-folded acoustic vibrations of first and second order in GaAs/AlAs superlattices.

Most time resolved experiments on coherent lattice excitations dealt with longitudinal optical phonons [6] or phonon polaritons [7]. While the excitation of phonon polaritons relies on impulsive stimulated Raman scattering (ISRS) [8], in the case of longitudinal optical phonons, a variety of excitation processes have been identified which cannot be explained within the context of stimulated
Raman scattering. The most prominent non-Raman type mechanisms are the displacive excitation of coherent phonons relevant for symmetry maintaining optical modes [9] and the generation of coherent LO phonons via rapid surface field screening in polar semiconductors [10]. One important hint towards the determination of the excitation process is the wave vector of the excited mode. Because of the flat dispersion of optical phonons in the vicinity of the BZ center their wave vector cannot be determined with high accuracy. However, due to the steeper dispersion of the acoustic modes, their wave vector can be determined accurately from the observed frequency, provided a high resolution and an accurate theoretical dispersion. Thus Raman and non-Raman excitation mechanisms can be distinguished.

The vibrational properties of the superlattice are analyzed within an elastic continuum model $[3,11]$, which yields an implicit equation for the acoustic dispersion in the low frequency limit $(<2 \mathrm{THz})$. The eigendisplacement fields are calculated by a Fourier expansion method which gives the symmetry properties of the vibrations and scattering intensities of the modes [4]. The lowest order dispersion reads

$$
\omega_{p, q}^{2}=\frac{C_{0}}{\rho_{0}}(q+p G)^{2}
$$

where $C_{0}$ and $\rho_{0}$ are the zeroth order expansion coefficients of the elastic stiffness constant $C_{11}$ and the crystal density along the growth direction and $q$ is the phonon wave vector. $G=2 \pi / d_{\mathrm{SL}}=2 \pi /\left(d_{\mathrm{GaAs}}+\right.$ $\left.d_{\mathrm{A} 1 \mathrm{As}}\right)$ is the reciprocal superlattice vector and $|p|(p=$ $0, \pm 1, \pm 2, \ldots)$ denotes the order of a folded acoustic vibration. Sound velocities and crystal densities are taken from Ref. [2]. In the investigated (001)-oriented samples the ratio of the AlAs layer thickness to the superlattice period $x=d_{\mathrm{AlAs}} /\left(d_{\mathrm{GaAs}}+d_{\mathrm{AlAs}}\right)$ is below 0.55 ; hence the displacement field of the BZ-center mode on the 
$p=-1$ branch has $A_{1}$ symmetry within the superlattice point group (antisymmetric with respect to a midplane of a layer). The mode with $p=+1$ has $B_{2}$ symmetry (symmetric). In a sample with $x=0.5$ the symmetry properties of the two second order BZ-center modes change from $A_{1}$ to $B_{2}$ and vice versa. At typical backscattering $q$ values of $2 \times k_{\text {laser }} \approx 0.2 \pi / d_{\text {SL }}$ symmetric and antisymmetric components are mixed [4]. If a Raman scattering process is the driving force of the lattice vibrations, only longitudinal acoustic modes with antisymmetric contributions to the displacement field are scattering active [4]. Transverse modes are forbidden for (001) surfaces. As a consequence at the $\mathrm{BZ}$ center the longitudinal antisymmetric modes are driven selectively, while at backscattering wave vector a doublet corresponding to the two branches of a folded order is active.

Three samples grown on a (001)-oriented GaAs substrate consisting of 40 periods are investigated. The parameters of the active zones are for sample $A$ : 19 monolayers (ML) GaAs and 19 ML AlAs (19/19), sample $B:(24 / 4)$, and sample $C:(18 / 5)$. Between the superlattice and the substrate an $\mathrm{Al}_{0.36} \mathrm{Ga}_{0.64} \mathrm{As}$ layer of $1 \mu \mathrm{m}$ is employed which is used as etch stop when removing the substrate for transmission experiments by wet etching.

Femtosecond time resolved investigations are performed in a standard pump-probe setup. An intense pump pulse derived from a tunable mode locked Ti:sapphire laser, tuned to the first interband transition of the samples, is focused to a spot of $\approx 120 \mu \mathrm{m}$ on the sample. The excitation density corresponds to a bulk excitation density of $10^{18}$ electron-hole pairs $/ \mathrm{cm}^{3}$. The pulse duration of approximately $50 \mathrm{fs}$ is far below the period of the first and second order folded acoustic phonons. A probe pulse of lower intensity, focused to a spot of $\approx 40 \mu \mathrm{m}$, is used to observe reflectivity and transmission changes as a function of time delay between pump and probe pulse. The reflectivity/transmission measurements are performed either with the full spectrum of the laser pulses or with a probe pulse, whose spectrum is narrowed by bandpass filters. For increasing the signal-to-noise ratio we measure the time resolved signal with a commercial data acquisition system based on a rapid scanning delay line (Aixscan, GWU Lasertechnik) which allows the detection of relative signal changes of some $10^{-7}$ without using a lock-in amplifier [10].

The reflectivity changes are dominated by carriers excited resonantly at the first interband transition. An initial reflectivity change in the order of $\Delta R / R_{0} \approx$ $10^{-3}$ is induced (inset in Fig. 1). The slowly decaying electronic signal is modulated by minute oscillations of the coherently generated acoustic phonons with an amplitude of $\Delta R / R_{0} \approx 10^{-5}$. In order to separate the lattice dynamics from the carrier dynamics, we extract the oscillating signature numerically. Frequency spectra are obtained by numerical Fourier transforms.

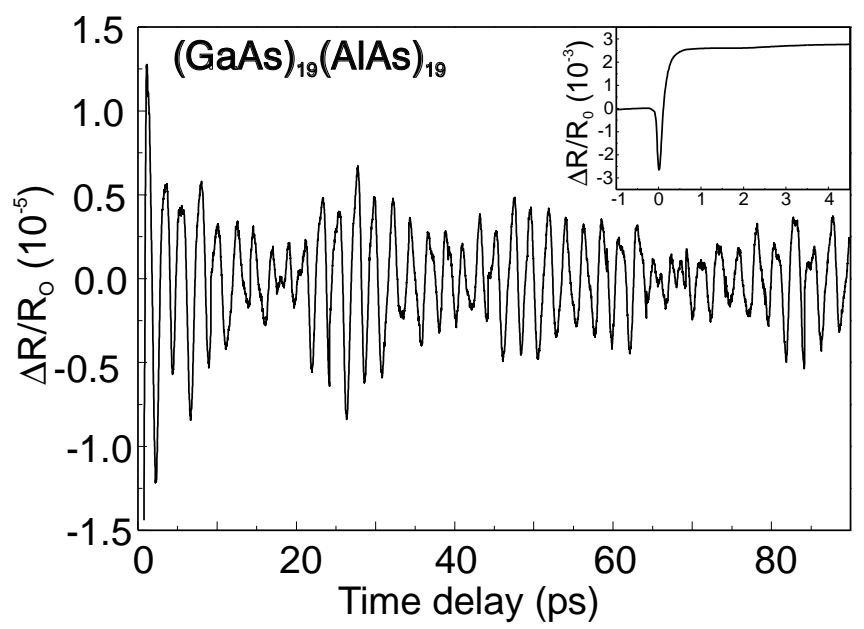

FIG. 1. Oscillatory transient reflectivity changes in a (19/19) superlattice. The inset shows the reflectivity changes.

Figure 1 shows the extracted spectrally integrated transient reflectivity changes induced by the excited coherent acoustic phonons in sample $A$ at room temperature. The signal exhibits a strong beating of several modes. The Fourier transform of the time resolved signal (solid line) and the calculated dispersion are depicted Fig. 2. The spectrum yields three modes of first and second order folded acoustic phonons. In order to identify the wave vectors we compare the spectrum to the calculated dispersion curve. The $q$ vectors of the modes $I_{1}, I_{2}$ and $I I I_{1}, I I I_{2}$ accurately match the backscattering $k$-vector transfer $q=$ $\Delta k_{\mathrm{BS}}=4 \pi n / \lambda$, where the refractive index $n$ is averaged over one superlattice period and $\lambda=797 \mathrm{~nm}$ is the central wavelength of the laser. This points towards ISRS in a backscattering direction as an excitation mechanism for these modes. At the backscattering $q$ value both branches of the same order are excited due to the mixing of

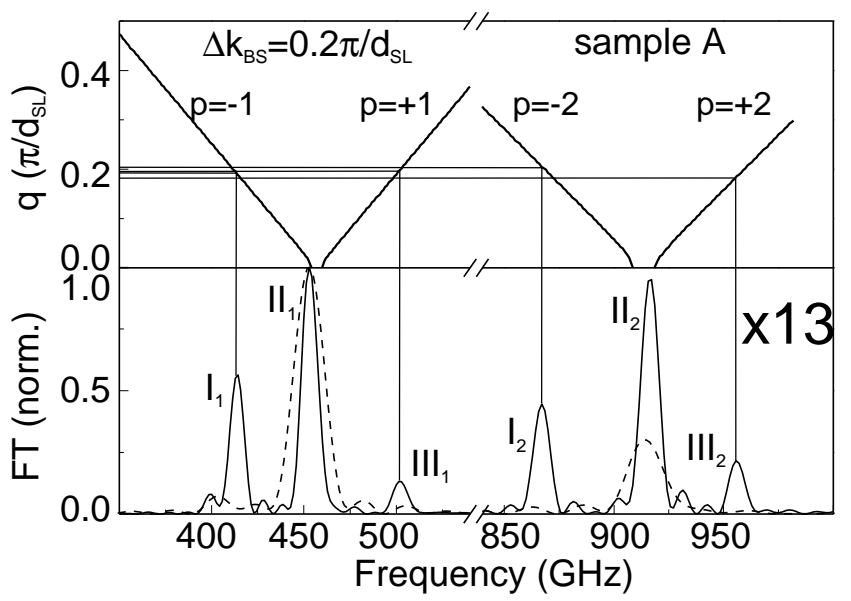

FIG. 2. Top: Dispersion of sample A. Bottom: Fourier power spectrum of the data shown in Fig. 1 (solid line) and of transmission changes (dashed line). The high frequency part is scaled by a factor of 13 . 
symmetric and antisymmetric properties of the displacement field. The central modes $I I_{1}$ and $I I_{2}$ are assigned to the $A_{1}$ zone center modes with $p=-1$ and $p=+2$ excited via forward scattering ISRS. This is evident from the good agreement between the spectrum and the dispersion and in accordance with the predictions of the Fourier expansion method on the Raman activity of the BZ center modes.

In transmission experiments on the same sample further evidence is gained that the peaks labeled $I I$ belong to BZ center modes. Under identical excitation conditions no triplet appears. Only the central frequencies $\left(I I_{1}, I I_{2}\right)$ are observed (Fig. 2, dashed spectrum). This is due to the fact that the total thickness of the superlattice is 4 times the wavelength of the modes with $q=2 \times k_{\text {laser }}$, averaging out signatures of the modes $I$ and $I I I$ in contrast to reflectivity measurements that are more sensitive towards the top layers of the superlattice.

The dispersion close to the center of the mini-BZ for each backfolded order is symmetric around the frequency $\omega_{p, q=0}^{2}=C_{0} / \rho_{0} \times(p G)^{2}$ at $q=0[12]$. The frequency difference $\left(\nu_{I I I}-\nu_{I I}\right)-\left(\nu_{I I}-\nu_{I}\right)$ is therefore equal to the forbidden gap. A positive (negative) value indicates, that the gap lies between $I I$ and $I I I$ ( $I$ and $I I$ ). For the first-order modes it is positive, and we thus confirm that the $I I_{1}$ peak must belong to the lower branch of $A_{1}$ symmetry $(p=-1)$. In second order it is negative and therefore mode $I_{2}$ belongs to the upper branch of second order with $p=+2$. Thus the prediction of the Fourier expansion method is confirmed that in a superlattice with $d_{\mathrm{AlAs}} /\left(d_{\mathrm{GaAs}}+d_{\mathrm{AlAs}}\right)=0.5$ the symmetry properties of the upper and lower branches of first- and second-order interchange.

The results obtained in sample $A$ are confirmed in samples $B$ and $C$, though only first-order phonons are resolved [13]. The modes $I_{1}$ and $I I I_{1}$ match the backscattering light wave vector transfer and the central modes $I I_{1}$ correspond to the $A_{1}$-symmetry BZ-center modes (Fig. 3). The gaps of first order in samples $B$ and $C$ are found to be smaller than the gaps obtained from the model calculation, treating the superlattice as abruptly layered continuum. The ratio of the experimental gap to the calculated gap is 0.5 for sample $B$ and 0.47 for sample $C$ in contrast to sample $A$ where no deviation of the experimental gap from the model gap is found. The size of the gap is proportional to the mismatch of the acoustic impedances

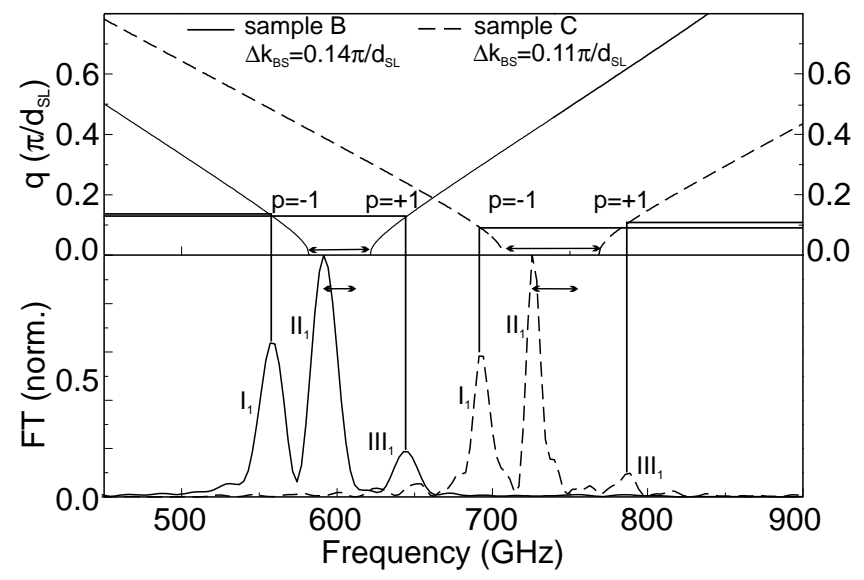

FIG. 3. Dispersion relation and experimental acoustic phonon spectra obtained in reflectivity experiments of samples $B$ and $C$. The arrows in the dispersion (spectrum) visualize the model (experimental) acoustic gap in the samples.

of the two constituting materials at the interfaces [12]. Smeared out interfaces due to monolayer fluctuations result in a more alloylike material and a reduced mismatch of the acoustic impedance. In contrast to sample $A$, in samples $B$ and $C$ the AlAs layers consist of only 4 and $5 \mathrm{ML}$, respectively. The deviation of the experimentally found gaps from the model calculation for these samples is attributed to the relatively larger influence of ML fluctuations at these small numbers of ML. Nonperfect interfaces do not affect the dispersion at backscattering $q$ values, because apart from the center and the edge of the mini-BZ the acoustic dispersion is well defined by folding of an averaged bulk dispersion [Eq. (1)].

We compare the observed Fourier powers of the first order modes $I_{1}$ and $I I I_{1}$ normalized to the $I I_{1}$ peak to the normalized calculated Raman scattering intensities (calculation according to Ref. [4]) in Table I. This comparison is possible since for both $\mathrm{cw}$ scattering and impulsive scattering the phonon-light interaction is mediated through the modulation of the photoelastic properties along the growth direction $[4,6]$, i.e., the same Raman-tensor elements. The experimental and calculated relative intensities agree well thus confirming ISRS as the driving mechanism.

In order to get information on the detection process, we perform energy resolved reflectivity and transmission experiments on sample $A$. The central wavelength of

TABLE I. Experimental Fourier powers (FP) of the modes $I_{1}$ and $I I I_{1}$, normalized to the power of the $I I_{1}$ peak, compared to the normalized calculated scattering intensities (int.) for samples $A, B$, and $C$.

\begin{tabular}{ccccccc}
\hline \hline & \multicolumn{2}{c}{ Sample $A$} & \multicolumn{2}{c}{ Sample $B$} & \multicolumn{2}{c}{ Sample $C$} \\
\hline Peak & Expt FP & Calc int. & Expt FP & Calc int. & Expt FP & Calc int. \\
$I_{1}$ & 0.56 & 0.55 & 0.64 & 0.63 & 0.58 & 0.68 \\
$I I_{1}$ & 0.13 & 0.45 & 0.2 & 0.36 & 0.1 & 0.29 \\
\hline \hline
\end{tabular}


the pump beam is tuned to the first interband transition of the sample at $1.548 \mathrm{eV}$ as determined by $\mathrm{cw}$ transmission spectroscopy. The probe beam is spectrally filtered behind the sample by a tunable bandpass filter of $10 \mathrm{meV}$ width. Figure 4 shows the amplitude of the observed phonon induced reflectivity changes as a function of the detection photon energy at constant pump energy. A strong resonant behavior in the vicinity of the interband resonance with a $\pi$ phase shift on either side is observed. No vibrations are detected at energies below $1.482 \mathrm{eV}$ and above $1.591 \mathrm{eV}$. Assuming that the reflectivity $R$ is mainly determined by changes of the refractive index $n$ rather than by changes in the extinction coefficient $k$, we can write

$$
\Delta R=\frac{\partial R}{\partial n} \frac{\partial n}{\partial E} \frac{\partial E}{\partial Q} \Delta Q .
$$

$\partial E / \partial Q$ is the deformation potential induced shift of the electron (hole) energy induced by the atomic displacement $\Delta Q$. Since we probe transitions close to the $\mathrm{BZ}$ center, we assume that at the discussed energies the dispersion of the deformation potential is negligible compared to the energy dependence of the reflectivity change amplitude. In the vicinity of a strong absorption peak the refractive index and thus $\partial n / \partial E$ has a schematical behavior as shown in the inset in Fig. 4. A comparison of the experimental reflectivity change amplitude as a function of the detection energy $\Delta R_{0}(E)$ with the schematic $\partial n / \partial E$ behavior indicates that $\partial n / \partial E$ is the dominating contribution to the energy dependence of the reflectivity amplitude.

In energy resolved experiments in transmission geometry where $\partial k / \partial E$ is the counterpart to $\partial n / \partial E$ we find exactly one $\pi$ phase shift within the range where the acoustic phonons can be detected at the first heavy holeelectron transition. This is in agreement with the expected $\partial k / \partial E$ behavior.

In conclusion we impulsively generated coherent zonefolded acoustic phonon modes of first and second order in GaAs/AlAs superlattices by resonant impulsive stimulated Raman scattering in forward and backward scattering directions. The obtained spectra are in good agreement with calculated dispersion curves, symmetry properties, and relative Raman scattering amplitudes. The modulation amplitude of the optical properties by the acoustic vibrations exhibits a strong resonant behavior as a function of the detection photon energy in the vicinity of the first interband transition.

This work is supported by the Deutsche Forschungsgemeinschaft. A. Bartels thanks the Konrad-Adenauer Stiftung for financial support.

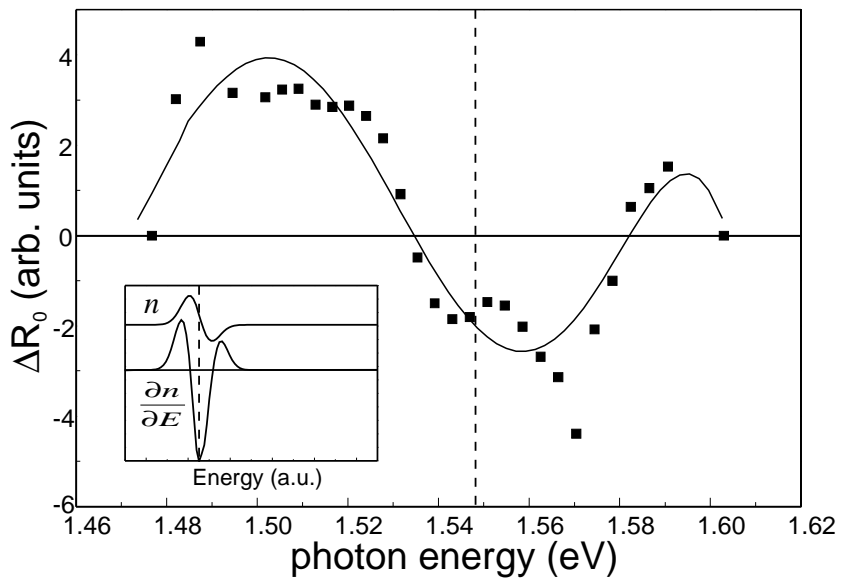

FIG. 4. Amplitude of the phonon induced reflectivity change in sample $A$ as a function of the detection energy. The inset depicts schematically the dispersion of $n$ and $\partial n / \partial E$ at an interband resonance. The dashed line in the figure marks the first heavy-hole to electron transition. The solid line is a guide to the eye.

Note added in proof. - Lately we noticed that coherent zone-folded acoustic phonons in GaAs/AlAs superlattices have been observed independently by two groups [14]. Their observations corroborate the conclusions reported here.

*Email address: bartels2@iht-ii.rwth-aachen.de

[1] J. J. Baumberg et al., Phys. Rev. Lett. 78, 3358 (1997).

[2] C. Colvard et al., Phys. Rev. Lett. 45, 298 (1980).

[3] C. Colvard et al., Phys. Rev. B 31, 2080 (1985).

[4] B. Jusserand et al., Phys. Rev. B 35, 2808 (1987).

[5] A. Yamamoto et al., Phys. Rev. Lett. 73, 740 (1994).

[6] For reviews, see, e.g., W. Kütt et al., IEEE J. Quantum Electron. 28, 2434 (1992); R. Merlin, Solid State Commun. 102, 207 (1997).

[7] H. Bakker et al., Rev. Mod. Phys. 70, 523 (1998).

[8] Y.X. Yan et al., J. Chem. Phys. 83, 5391 (1985).

[9] T. K. Cheng et al., Appl. Phys. Lett. 59, 1923 (1991).

[10] G. C. Cho et al., Phys. Rev. Lett. 65, 764 (1990).

[11] S. M. Rytov, Akust. Zh. 2, 71 (1956) [Sov. Phys. Acoust. 2, 68 (1956)].

[12] B. Jusserand and M. Cardona, in Light Scattering in Solids (Springer, Berlin, 1989), Vol. V, p. 49.

[13] The sensitivity towards higher order modes can be enhanced by multiple pulse excitation [A. Bartels et al., Appl. Phys. Lett. 72, 2844 (1998)].

[14] K. Mizoguchi et al., Physica (Amsterdam) 249B-251B, 887 (1998); T. Mishina et al., Solid State Commun. 107, 281 (1998). 